\title{
The solar-like CoRoT target HD 170987: spectroscopic and seismic observations ${ }^{\star}$
}

\author{
S. Mathur ${ }^{1,2}$, R. A. García ${ }^{1}$, C. Catala ${ }^{3}$, H. Bruntt ${ }^{3}$, B. Mosser $^{3}$, T. Appourchaux ${ }^{4}$, J. Ballot ${ }^{5}$, O. L. Creevey ${ }^{6,7}$

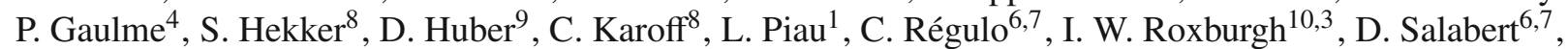 \\ G. A. Verner ${ }^{10}$, M. Auvergne ${ }^{3}$, A. Baglin ${ }^{3}$, W. J. Chaplin ${ }^{8}$, Y. Elsworth ${ }^{8}$, E. Michel $^{3}$, R. Samadi ${ }^{3}$, \\ K. Sato ${ }^{1}$, and D. Stello 9 \\ 1 Laboratoire AIM, CEA/DSM - CNRS, Université Paris Diderot - IRFU/SAp, 91191 Gif-sur-Yvette Cedex, France \\ e-mail: savita.mathur@gmail.com \\ 2 High Altitude Observatory, NCAR, PO Box 3000, Boulder, CO 80307, USA \\ 3 LESIA, UMR8109, Université Pierre et Marie Curie, Université Denis Diderot, Obs. de Paris, 92195 Meudon Cedex, France \\ ${ }^{4}$ Institut d'Astrophysique Spatiale, UMR 8617, Université Paris XI, Bâtiment 121, 91405 Orsay Cedex, France \\ 5 Laboratoire d'Astrophysique de Toulouse-Tarbes, Université de Toulouse, CNRS, 31400 Toulouse, France \\ ${ }^{6}$ Universidad de La Laguna, Dpto de Astrofísica, 38206, Tenerife, Spain \\ 7 Instituto de Astrofísica de Canarias, 38205, La Laguna, Tenerife, Spain \\ 8 School of Physics and Astronomy, University of Birmingham, Edgbaston, Birmingham B15 2TT, UK \\ 9 Sydney Institute for Astronomy, School of Physics, University of Sydney, NSW 2006, Australia \\ 10 Astronomy Unit, Queen Mary University of London, Mile End Road, London E1 4NS, UK
}

Received 19 January 2010 / Accepted 28 April 2010

\section{ABSTRACT}

\begin{abstract}
Context. The CoRoT mission is in its third year of observation and the data from the second long run in the galactic centre direction are being analysed. The solar-like oscillating stars that have been observed up to now have given some interesting results, specially concerning the amplitudes that are lower than predicted. We present here the results from the analysis of the star HD 170987.

Aims. The goal of this research work is to characterise the global parameters of HD 170987. We look for global seismic parameters such as the mean large separation, maximum amplitude of the modes, and surface rotation because the signal-to-noise ratio in the observations does not allow us to measure individual modes. We also aim to retrieve the parameters of the star and its chemical composition.

Methods. We studied the chemical composition of the star through ground-based observations performed with the NARVAL spectrograph. We used several methods to calculate the global parameters from the acoustic oscillations based on CoRoT data. The light curve of the star has been interpolated with inpainting algorithms to reduce the effect of data gaps.

Results. We found the power excess related to p modes in the range [400-1200] $\mu \mathrm{Hz}$ with a mean large separation of $55.2 \pm 0.8 \mu \mathrm{Hz}$ with a probability above $95 \%$ that increases to $55.9 \pm 0.2 \mu \mathrm{Hz}$ in a higher frequency range [500-1250] $\mu \mathrm{Hz}$ and a rejection level of $1 \%$. A hint of the variation of this quantity with frequency was also found. The rotation period of the star is estimated to be around 4.3 days with an inclination axis of $i=50^{\circ}{ }_{-13}^{+20}$. We measured a bolometric amplitude per radial mode in a range [2.4-2.9] ppm around $1000 \mu \mathrm{Hz}$. Finally we estimate the stellar mass with a grid of models, $M=1.43 \pm 0.05 M_{\odot}$, the radius, $R=1.96 \pm 0.046 R_{\odot}$, and the age $\sim 2.4 \mathrm{Gyr}$.
\end{abstract}

Key words. asteroseismology - methods: data analysis - stars: oscillations - stars: individual: HD 170987

\section{Introduction}

During the present decade the number of confirmed solar-like pulsators - those with acoustic modes excited by turbulent motions in the near-surface convection (e.g. Christensen-Dalsgaard 2004, and reference therein) - has increased enormously thanks first to the growing number of ground-based observing campaigns (e.g. Bedding \& Kjeldsen 2007; Arentoft et al. 2008), and second to the high-precision photometry measurements provided by space instrumentation such as WIRE (Wide-Field Infrared Explorer, e.g. Bruntt et al. 2007), MOST (Microvariability and Oscillations of STars, Walker et al. 2003), and CoRoT

* The CoRoT space mission, launched on 2006 December 27, has been developed and is operated by CNES, with the contribution of Austria, Belgium, Brazil, ESA (RSSD and Science Programme), Germany and Spain.
(Baglin et al. 2006). The latter has been providing data with an unprecedented quality both in terms of photometric precision and in terms of uninterrupted observation lengths. CoRoT has already observed several main-sequence solar-like pulsators (Michel et al. 2008) while it has enabled us to resolve the individual modes of the oscillations spectra of several $\mathrm{F}$ stars (Appourchaux et al. 2008; Barban et al. 2009; García et al. 2009; Mosser et al. 2009b) and G stars (Ballot et al., in prep.; Deheuvels et al. 2010). At least, it allowed us to derive a large spacing for the faintest targets (García et al. 2009; Mosser et al. $2009 b)$. The measurement of these seismic parameters already offer a valuable tool for accurate determinations of radii (e.g. Stello et al. 2009) and ages (e.g. Creevey 2008) of stars, which are particularly interesting for a better understanding of stellar evolution as well as for the characterisation of stars hosting planets. All these CoRoT observations are the starting point for a 


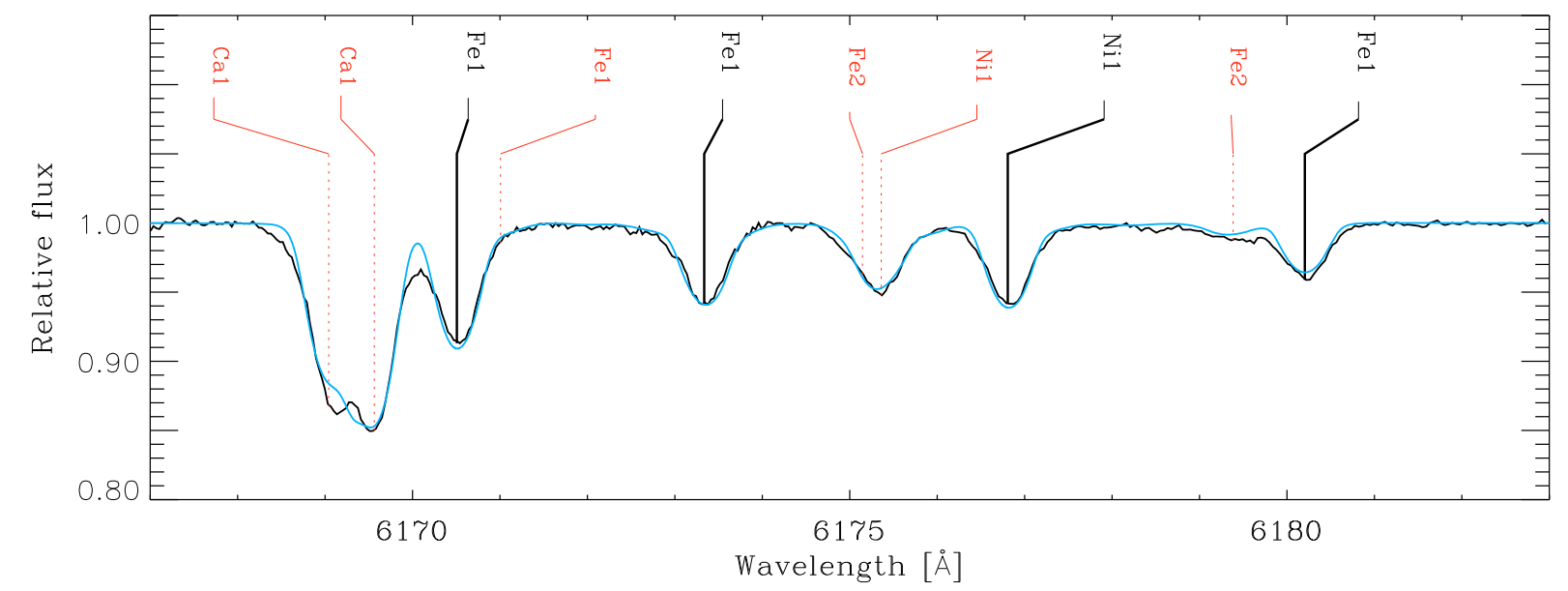

Fig. 1. Comparison of the observed (black) and computed (blue) spectrum of HD 170987. The four solid vertical lines mark the lines used in the spectral analysis, while the vertical dotted lines show other well-known lines.

better understanding of the structure (Piau et al. 2009; Deheuvels \& Michel 2009) and the surface dynamics (Ludwig et al. 2009; Samadi et al. 2010a,b) of this class of stars.

The Kepler mission, successfully launched on 2009 March 7 (Borucki et al. 2009), will also contribute to this field by observing stars on very long runs (four years). The quality of the first data on stars with solar-like oscillations (Bedding et al. 2010a; Chaplin et al. 2010; Hekker et al. 2010b; Stello et al. 2010) promises the asteroseismology a bright future for the study of stellar interiors and dynamical processes (ChristensenDalsgaard \& Houdek 2009; Samadi 2009; Mathis 2009).

We present results about a star recently observed by CoRoT, HD 170987 (or HIP 90851). This target is a well-known double star where components are separated by $0.7^{\prime \prime}$ (e.g. Dommanget $\&$ Nys 2002). The main star is a F5 dwarf star with a magnitude $m_{V}$ ranging from 7.4 to 7.7 in the literature, while the second component has a magnitude around 8.5.

We start by reporting the latest spectroscopic results observed by the NARVAL spectrograph (in Sect. 2), which shows that this star is very similar to Procyon (e.g. Allende Prieto et al. 2002). Then we describe the observations done with CoRoT during 149 days and the interpolation done in the data gaps of the light curve (Sect. 3). In Sect. 4 we infer the surface-rotation period of the star from the detailed analysis of the low-frequency region of the power spectrum and then we obtain the global properties of the acoustic modes and of the star in Sects. 5 and 6 respectively. We finish in Sect. 7 with a discussion of the results and in Sect. 8 with the conclusions of the paper.

\section{Spectroscopic results}

We observed HD 170987 with the NARVAL spectrograph on the 2-m class Bernard Lyot Telescope at the Pic du Midi Observatory. We acquired one spectrum on each night of 2009 July 7, 810,11 and 13 . The spectra were co-added to obtain a signal-to-noise ratio in the continuum of $S / N \approx 900$ in the range 5800-6400 A. As an example of the quality of the spectrum, a small section is shown in Fig. 1.

We analysed the spectrum with the semi-automatic software package VWA (Bruntt et al. 2004; Bruntt 2009), which uses atmospheric models interpolated in the MARCS grid (Gustafsson et al. 2008) and atomic parameters from VALD (Kupka et al. 1999). The abundances for 239 lines were calculated iteratively by fitting synthetic profiles to the observed spectrum using
SYNTH (Valenti \& Piskunov 1996). For each line the abundances were differentially calculated with respect to the same line in a solar spectrum from Hinkle et al. (2000). The atmospheric parameters $\left(T_{\text {eff }}, \log g,[\mathrm{Fe} / \mathrm{H}]\right.$, and $\left.v_{\text {micro }}\right)$ were determined by requiring that $\mathrm{Fe}$ lines gave the same abundance independent of their equivalent width $(E W)$, excitation potential (EP) or ionization stage. Only weak lines were used $(E W<90 \mathrm{~m} \AA)$ for this part of the analysis as they are sensitive to adjust the parameters, while stronger lines $(E W<140 \mathrm{~m} \AA)$ were used for the calculation of the final mean abundances. The uncertainties on the atmospheric parameters and the abundances were determined by perturbing the best-fitting atmospheric parameters, as described by Bruntt et al. (2008).

For F-type stars like HD 170789, non-LTE (local thermodynamical equilibrium) effects cannot be ignored. Our model of atmospheres assumes LTE and we therefore systematically underestimate the abundance of Fe I while Fe II is nearly unaffected. We followed the approach of Bruntt et al. (2008) and corrected the Fe I abundance with the results of Rentzsch-Holm (1996). The effect is $[\mathrm{Fe} \mathrm{I} / \mathrm{H}]_{\mathrm{NLTE}}=[\mathrm{Fe} \mathrm{I} / \mathrm{H}]_{\mathrm{LTE}}+0.046$ dex. This affects our estimate of the surface gravity $\log g$, as was discussed by Fuhrmann et al. (1997) in the case of Procyon, which has a very similar spectral type. Following their approach we used the Mg I b lines at 5173 and $5184 \AA$ to determine $\log g$. We first adjusted the Van der Waals broadening constants from VALD to fit the solar spectrum for the canonical value of $\log g=4.437$ dex. We then determined the $\mathrm{Mg}$ abundance in HD 170987 from two weak $\mathrm{Mg}$ lines and fitted the wings of the two strong $\mathrm{Mg} \mathrm{I} \mathrm{b}$ lines. An example of the fit is shown in Fig. 2, where the observed spectrum is compared to two synthetic spectra with $\log g=4.35$ and 3.95 dex. The hatched regions near the border of the plot are used to normalise the spectrum and the grey-shaded rectangles show the sections of the spectrum used to compute the $\chi^{2}$ value needed to determine the best value of $\log g$. The mean value of the two $\mathrm{MgI}$ b lines gives $\log g=4.35 \pm 0.22$ dex. This value is slightly higher but agrees with the more precise value determined from the analysis of Fe I + II lines: $\log g=$ $4.20 \pm 0.05$ dex. Although the latter analysis is affected by our adopted correction for the NLTE effect, we use it as our final estimate of the surface gravity.

From our spectral analysis of HD 170987 we determine the following parameters: $T_{\text {eff }}=6540 \pm 36 \pm 70 \mathrm{~K}, \log g=$ $4.20 \pm 0.05 \pm 0.10 \mathrm{dex}$, and $v_{\text {micro }}=1.70 \pm 0.10 \pm 0.05 \mathrm{~km} \mathrm{~s}^{-1}$. We give two uncertainties for these values, the first is the intrinsic 


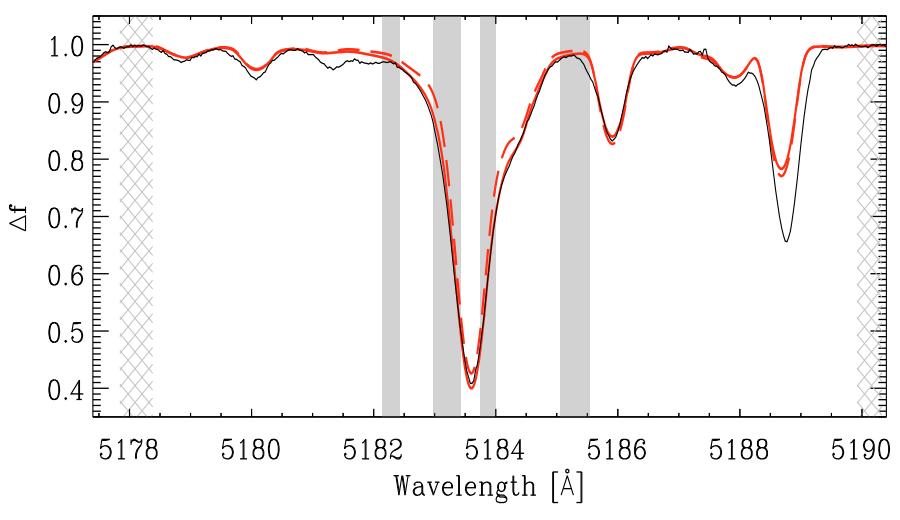

Fig. 2. Two synthetic profiles with different surface gravity are compared to the observed (black) spectrum $(\log g=4.35$ (solid line) and 3.95 dex (dashed line)). The hatched regions are used for renormalising the spectrum. The solid rectangles mark the regions where the $\chi^{2}$ is computed to determine the optimal value of $\log g$.

Table 1. Chemical composition of HD 170987 measured relative to the Sun $(\Delta A) . n$ is the number of spectral lines used.

\begin{tabular}{l|lr|lll}
\hline \hline El. & $\Delta A($ dex $)$ & $n$ & El. & $\Delta A($ dex $)$ & $n$ \\
\hline Li I & 1.80 & 1 & Ca I & $-0.10 \pm 0.03$ & 11 \\
C I & -0.27 & 2 & Sc II & $-0.16 \pm 0.06$ & 3 \\
O I & -0.02 & 1 & Ti I & $-0.15 \pm 0.05$ & 4 \\
Na I & -0.18 & 2 & Ti II & $-0.15 \pm 0.08$ & 2 \\
Mg I & -0.25 & 1 & Cr I & $-0.18 \pm 0.05$ & 7 \\
Mg II & -0.22 & 1 & Cr II & $-0.17 \pm 0.05$ & 5 \\
Al I & $-0.22 \pm 0.10$ & 4 & Fe I & $-0.15 \pm 0.03$ & 143 \\
Si I & $-0.19 \pm 0.03$ & 11 & Fe II & $-0.15 \pm 0.03$ & 20 \\
Si II & $-0.16 \pm 0.03$ & 2 & Ni I & $-0.27 \pm 0.03$ & 16 \\
S I & -0.27 & 2 & Y II & -0.10 & 1 \\
\hline
\end{tabular}

error and the second is the estimated systematic error. The systematic errors were estimated from a large sample of F5-K1 type stars (Bruntt et al. 2010). These two uncertainties must be added quadratically (e.g. $T_{\text {eff }}=6540 \pm 80 \mathrm{~K}$ ). For the mean metallicity we used the Fe II lines to get $[\mathrm{M} / \mathrm{H}]=-0.15 \pm 0.06$ dex. In Table 1 we list the mean abundances of 16 elements compared to the Sun (Grevesse et al. 2007), which are also shown in Fig. 3. Finally, for the projected rotational velocity we find $v \sin i=19.0 \pm 1.5 \mathrm{~km} \mathrm{~s}^{-1}$, which is significantly greater than for Procyon.

We also generated a mean profile from the spectrum using the least-squares deconvolution technique developed by Donati et al. (1997). This shows that the spectral lines are slightly asymmetric, indicating that the spectrum is contaminated by a fainter star. The determined spectroscopic parameters are only weakly affected by this.

In the catalogue of the Geneva-Copenhagen Survey of Solar neighbourhood III (Holmberg et al. 2007), based on Strömgren photometry, a value of $m_{V}=7.46$ was given. The new reduction of Hipparcos data (van Leeuwen 2007a,b) gives a parallax of $\pi=11.21 \pm 1.02$ mas, corresponding to a distance of $89 \pm 9 \mathrm{pc}$, leading to an absolute magnitude of $M_{V}=2.71$.

To get a first estimate of the seismic parameters of this star, we can use the value for the effective temperature found. The Hipparcos parallax combined with a bolometric correction of $-0.01 \pm 0.01$ derived using $T_{\text {eff }}$ in the calibration of Flower (1996) gives $L / L_{\odot}=6.41 \pm 1.2$ (neglecting interstellar reddening) and consequently $R / R_{\odot}=2.1 \pm 0.2$. A rough comparison with solar-metallicity evolutionary tracks gives a mass of $M / M_{\odot}=1.4 \pm 0.2$, which finally yields an estimate of the position of maximum amplitude, $\nu_{\max }=910 \pm 50 \mu \mathrm{Hz}$ and the mean large separation, $\Delta v \sim 52.7 \pm 4 \mu \mathrm{Hz}$ (Kjeldsen \& Bedding 1995). Compared to the other published CoRoT F stars (e.g. Barban et al. 2009; García et al. 2009), HD 170987 is therefore notably more similar to Procyon (e.g. Arentoft et al. 2008, and references therein).

\section{CoRoT photometric observations}

The star HD 170987 has been observed by CoRoT in the seismic field during 149 days starting on 2008 April 11 till 2008 September 7, during the second long run in the galactic centre direction (LRc02). In Fig. 4 we show the N2 light curve (in ppm) corrected for the ageing of the CCD by dividing by a third-order polynomial fit. We used timeseries that are regularly spaced in the heliocentric frame (i.e. the so-called Helreg level 2 data, Samadi et al. 2007a) with a 32 s sampling rate. The overall duty cycle of the timeseries is $89.7 \%$.

To compute the power spectrum density (PSD) we used a standard fast Fourier transform algorithm and we normalised it as the so-called one-sided power spectral density (Press et al. 1992). The resulting PSD is shown in Fig. 5.

The existing gaps are mostly due to the crossing of the South Atlantic Anomaly (SAA) ( for more details see Auvergne et al. 2009). The influence of the perturbations of SAA crossings is not completely attenuated by the standard correction procedure and thus yields alias peaks at multiples of the CoRoT orbital frequency of $161.7 \mu \mathrm{Hz}$ (vertical dotted lines in Fig. 5, top). For each of them a daily modulation can be observed at $\pm 11.57 \mu \mathrm{Hz}$ and at twice this frequency (see the inset of Fig. 5, top). In particular, the region where we expect to find the acoustic-mode power excess (centred around $900 \mu \mathrm{Hz}$ ) is completely dominated by this peak structure. Thus, we need to interpolate the data in the best way to reduce these effects as much as possible, which was already done for HD 175726 (Mosser et al. 2009b). For that star the algorithm proposed by Holdschneider et al. (1989) was used and gave excellent results. In our case, we adopted a different strategy based on the "inpainting" technique, which employs a multi-mcale discrete cosine transform (see Appendix A for a more detailed explanation of the algorithm). This algorithm was tested and the results were verified (for further details see Sato et al. 2010). Moreover, different groups have also used other interpolation techniques (linear, based on wavelets, etc.), leading to results for the global parameters of the modes (such as the mean large separation) that agree within the error bars.

\section{Surface rotation}

An independent measurement of the surface rotation can be obtained through the signature left by the active regions that cross the visible stellar disk. It provides a first estimate of the stellar rotation. Moreover, combined with the measured $v \sin i=$ $19.0 \pm 1.5 \mathrm{~km} \mathrm{~s}^{-1}$ (see Sect. 2) and assuming a given radius of the star, we can also estimate the inclination axis of the star, which can be very helpful to better constrain the fitting techniques to characterise the acoustic modes (Gizon \& Solanki 2003; Ballot et al. 2006, 2008).

The light curve of HD 170987 shows a periodic modulation of about four days (see Fig. 4), which could be due to the combination of the stellar magnetic activity (star spots) and the rotation of the star. It is interesting to analyse this periodicity in a more detailed way by studying the low-frequency range of its power spectrum. This is shown in Fig. 6, where a clear peak appears at 


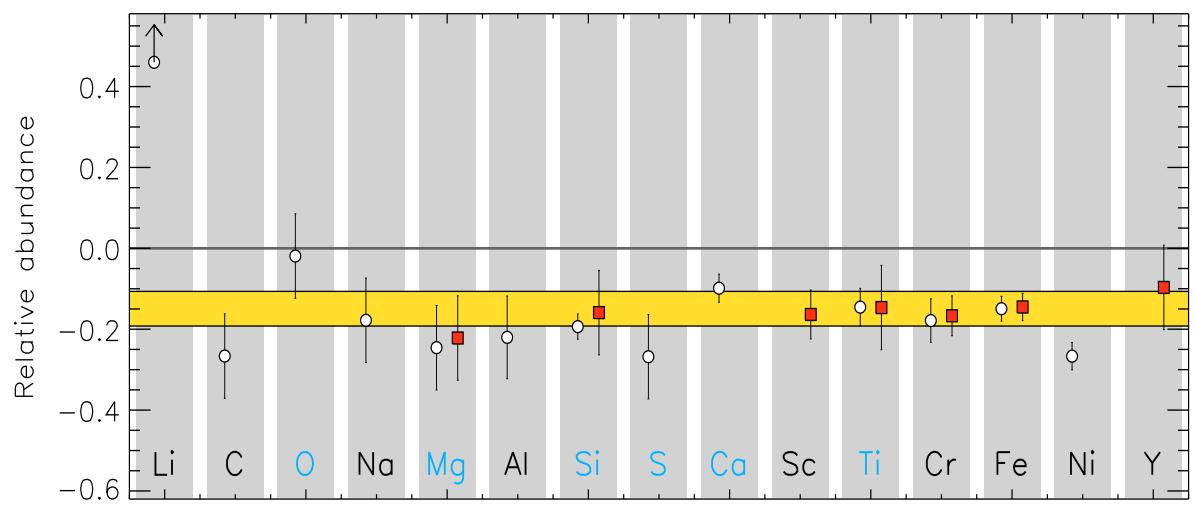

Fig. 3. Abundance pattern of HD 170987 for 16 elements. Circles and box symbols are used for the mean abundance from neutral and singly ionised lines, respectively. Notice the high abundance of lithium. The yellow horizontal bar marks the mean metallicity with $1-\sigma$ uncertainty range.

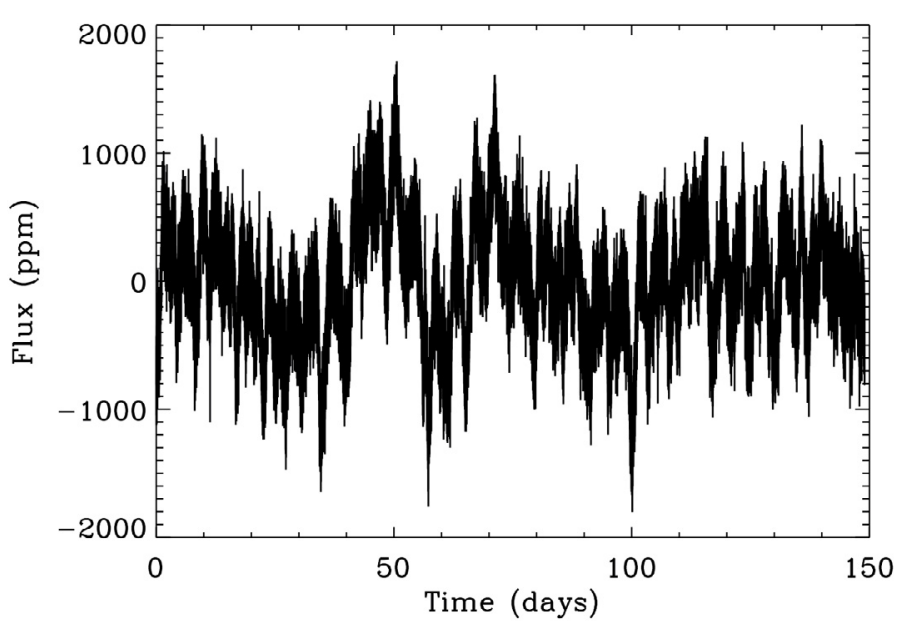

Fig. 4. N2-Helreg timeseries of the CoRoT target HD 170987 corrected from the ageing of the CCD. For plotting purposes only every fifth point is shown.

$2.70 \mu \mathrm{Hz}$. This frequency corresponds to a period of 4.3 days, which can be associated to the rotation period of the surface of the star.

At lower frequency - around $0.32 \mu \mathrm{Hz}$ - another structure appears, which could be related to low-frequency noise. Indeed, considering that the radius is estimated to $\sim 2 R_{\odot}$ and that the observed $v \sin i$ is of $\sim 19 \mathrm{~km} \mathrm{~s}^{-1}$, the second peak is very unlikely to be produced by the rotation of the stellar surface.

Spot-modelling of the light curve was performed according to the method introduced in Mosser et al. (2009a). Because all stars analysed in that paper, which have a spectral type close to HD 170987, show evidence of differential rotation, we used the fitting method D (Table 2 of Mosser et al. 2009a) with a fixed value of the differential rotation profile. This method has proven to be efficient for the determination of the rotation period and of the spot lifetime. Measurements of the star inclination and differential rotation are much less precise.

The best-fit model is presented in Fig. 7 for a 60-day long sample. Like for similar types of stars, the modelling is not able to reproduce the sharpest features of the light curve. Fitting them would require the introduction of too many spots, which lowers the global precision of the best-fit parameters. This fit is obtained for an inclination of $40_{-10}^{+20 \circ}$. The equatorial rotation period is $4.30 \pm 0.05$ days and the differential rotation rate $5_{-5}^{+10} \%$ for a mean rotational period close to $4.40 \mathrm{~d}$. The spot lifetime of $2.5_{-0.5}^{+1.0}$ days is significantly shorter than one rotation period of the star.

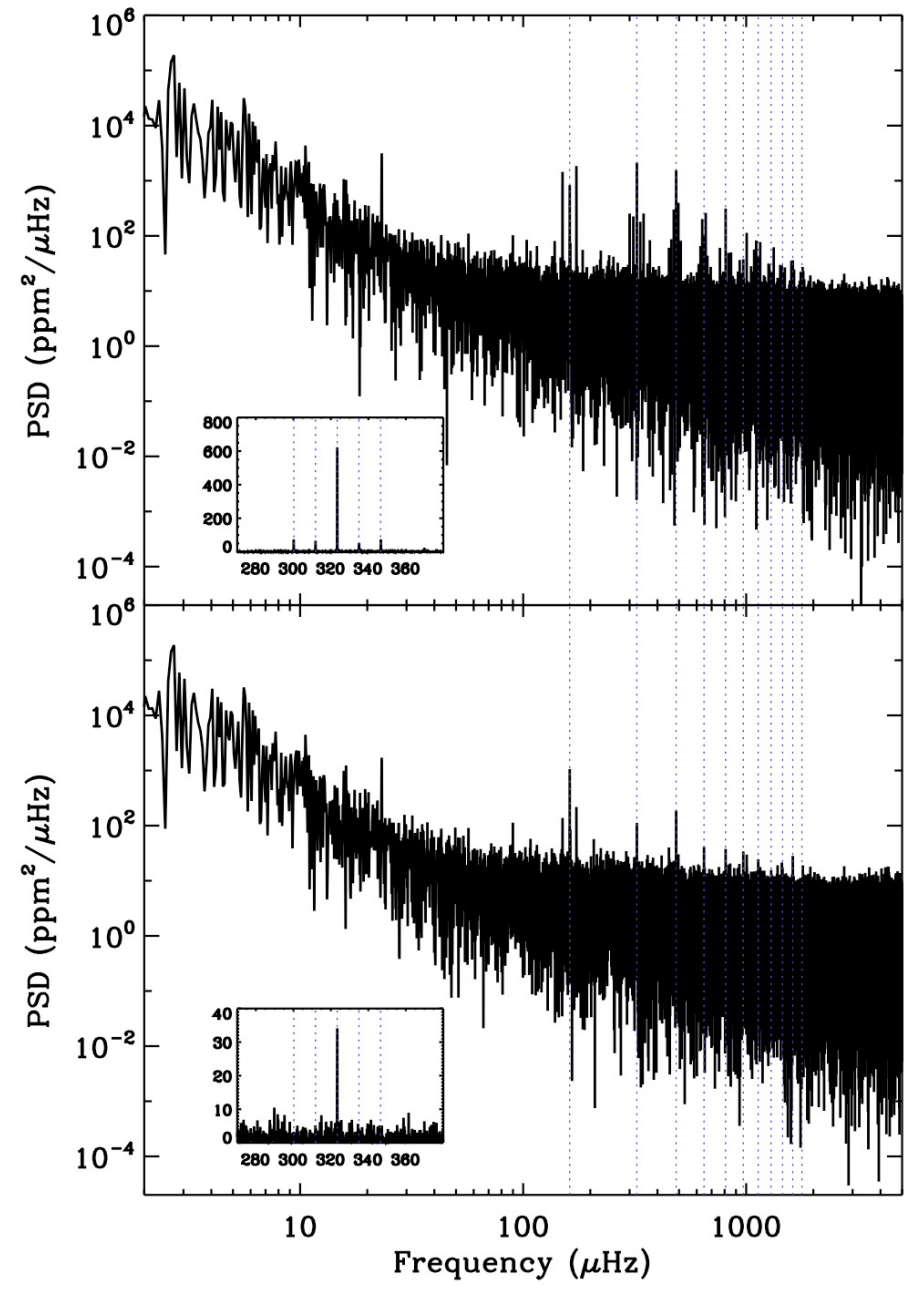

Fig. 5. Power spectrum density (in units of $\mathrm{ppm}^{2} / \mu \mathrm{Hz}$ ) of the raw N2Helreg timeseries (top) and after interpolating the data gaps by a multiscale discrete cosine transform as explained in the text (bottom). The vertical dotted lines represent the frequency of the CoRoT orbit at $161.7 \mu \mathrm{Hz}$ and the first 10 harmonics. The inserts are a zoom around the second orbital harmonic, normalised by its standard deviation, showing daily modulation with peaks at $\pm 11.57 \mu \mathrm{Hz}$ and at twice this frequency.

We used the wavelet technique to confirm the rotation period of the star's surface (Torrence \& Compo 1998). We used the Morlet wavelet (a moving Gaussian envelope with a varying width) to produce the wavelet power spectrum shown in Fig. 8. This spectrum represents the correlation between the wavelet with a given frequency ( $y$-axis) along time ( $x$-axis). As showed in Mathur et al. (2010), the strength of this method is to see the 


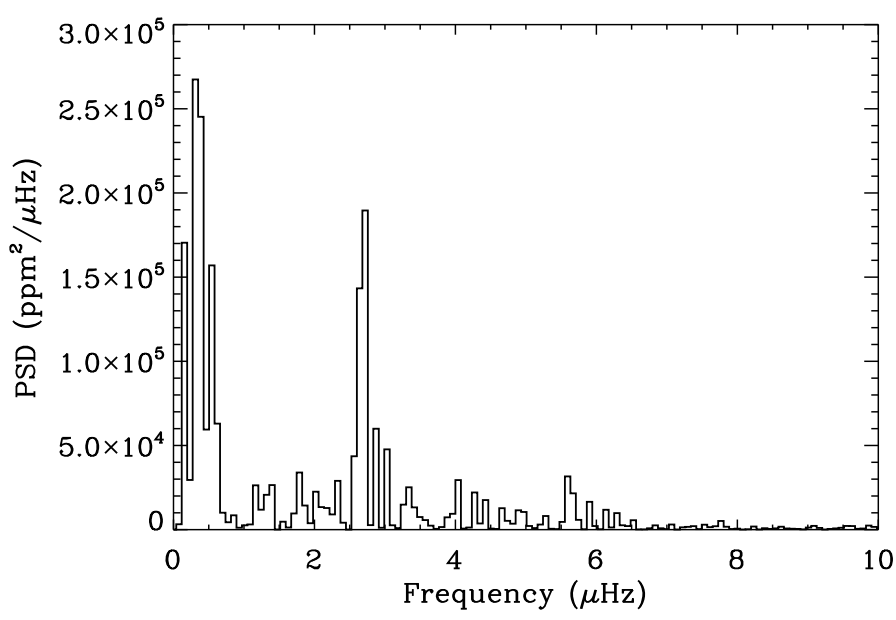

Fig. 6. Zoom on the low-frequency part of the PSD. The spectrum was computed with a classical FFT using the full timeseries.

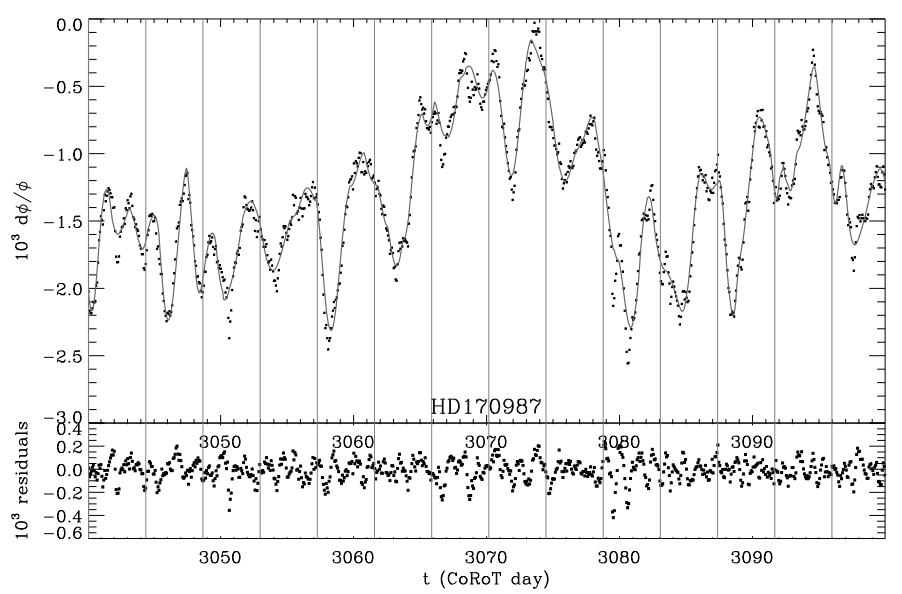

Fig. 7. Spot modelling of HD 170987. The dots in the upper panel represent the data binned every CoRoT orbit (6184 s) and the solid curve is the best-fit model. Dots in the bottom panel represent residuals. Vertical grey lines indicate the mean rotation period.

evolution with time of the power, as well as to resolve the uncertainty between the fundamental period and the first harmonic that could happen, as in the solar case (Mathur et al. 2008). The left panel of the plot shows the power in the wavelet spectrum for periods between four and five days. This power excess is observed for all the 149 days of observation. When collapsed over the observation time (right panel) we see the accumulation of power at 4.3 days well above the $95 \%$ confidence level limit.

The reliability of the result depends on two factors: the cone of influence, which is related to the fact that the periodicity has to be at least one quarter of the total length of the timeseries and a confidence level corresponding to a $95 \%$ of probability that the peak observed is not due to noise. With this in mind we determined that the rotation period of HD 170987 is $\sim 4.3$ days (or $2.7 \mu \mathrm{Hz}$ ), which is compatible with the low-frequency analysis of the PSD described above.

It is very clear that the power present around $2.7 \mu \mathrm{Hz}$ is concentrated between the 30th and the 70th day (black shaded region) as well as during another short period around the 100th day. This agrees with the results obtained with the PSD.

Given the value of the rotation period and the $v$ sin $i$, we can deduce the inclination angle $i=50^{\circ}{ }_{-13}^{+20}$.

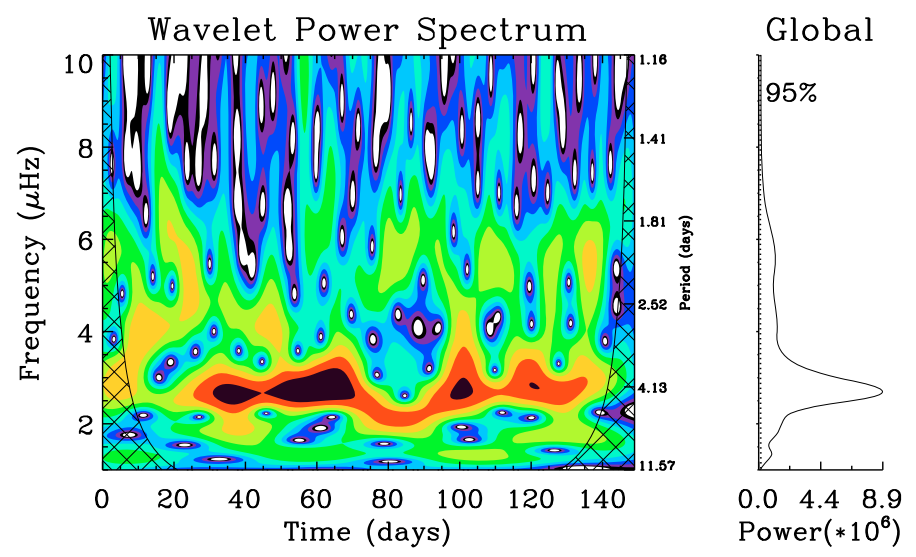

Fig. 8. Left panel: Wavelet power spectrum for HD 170987 as a function of the frequency of the Morlet wavelet ( $y$-axis) and time ( $x$-axis). The black grid represents the cone of influence. Right panel: Global power spectrum as a function of the frequency of the wavelet. The dotted line is the confidence level corresponding to a $95 \%$ probability.

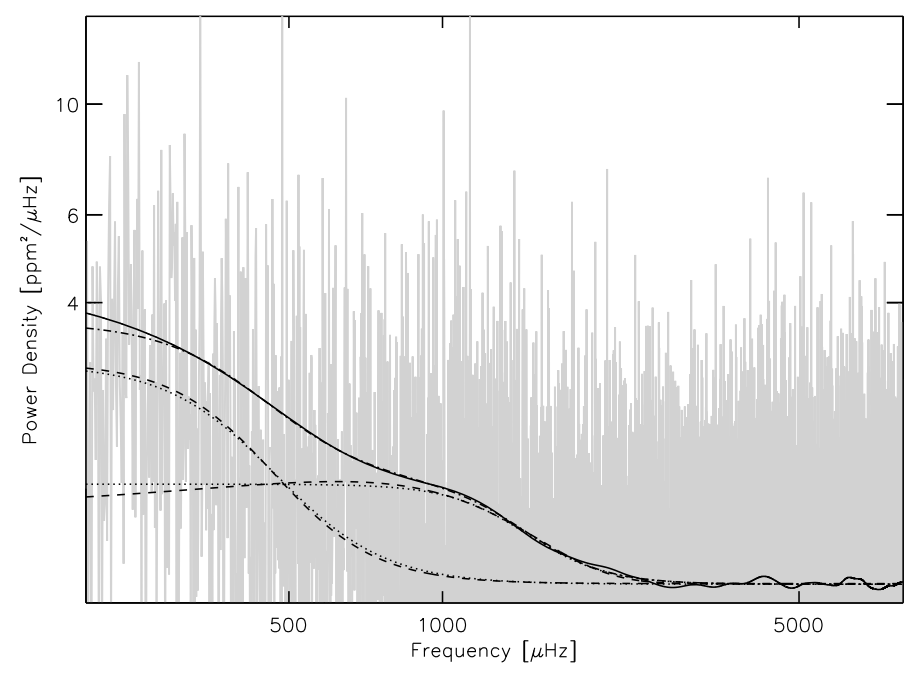

Fig. 9. Smoothed PSD (continuous solid line) overlaid by the background fitted model assuming two Harvey-model components (dotted lines) and a single Harvey model plus a p-mode excess (dashed lines). The individual components of the fitted background are also shown. The PSD have been smoothed with a Gaussian running mean with a width of $200 \mu \mathrm{Hz}$.

\section{Global parameters of the acoustic modes}

With different methods we estimated the global parameters of the acoustic modes of HD 170987, such as the location of the p-mode bump, the mean large spacing, and the maximum bolometric amplitude per radial mode.

\subsection{P-mode excess power in the PSD}

To evaluate the possible presence of p-mode oscillations in the spectrum we started by analysing a smoothed version of the PSD. We smoothed the PSD in the range 200 to $8000 \mu \mathrm{Hz}$ with a Gaussian running mean with a width of $200 \mu \mathrm{Hz}$. The reason why we did not include the low-frequency part of the spectrum is that this region of the PSD mainly bears the slow trend signatures in the data and of stellar activity (rotation), which was explained in Sect. 4. The smoothed PSD is shown in Fig. 9. Two clear bumps are seen in the spectrum, one around 300 and one around $1000 \mu \mathrm{Hz}$. The bump around $1000 \mu \mathrm{Hz}$ could 
originate from the p-mode oscillations (as it corresponds to the expected $v_{\max }$ ), but it could also be a signal related to granulation. Up to now, the background of all precedent CoRoT solarlike targets (HD 49933, HD 175726, HD 181420, HD 181906 and HD 49385) were correctly characterized by a single component in the Harvey model (Harvey 1985), which was associated to granulation. However, when comparing the spectrum of HD 170987 to that of the Sun obtained with the VIRGO instrument (Fröhlich et al. 1995) and to solar-type stars observed by the Kepler satellite (Chaplin et al. 2010), we see that in these cases a second Harvey power law has been used to take into account some extra power located around $1000 \mu \mathrm{Hz}$ (timescale close to $80 \mathrm{~s}$ ). Based on the smoothed PSD we can therefore not conclude that the spectrum shows p-mode oscillations, while we see some excess power centred at $\sim 1000 \mu \mathrm{Hz}$.

For the precise analysis of the acoustic background, different strategies were followed to study the bump at $1000 \mu \mathrm{Hz}$. We started by assuming that the bump around $300 \mu \mathrm{Hz}$ originates from granulation and that at $\sim 1000 \mu \mathrm{Hz}$ could originate from a shorter convective scale or by faculae.

The faculaes are the bright points seen in visual solar images often close to the dark sunspots. They are due to changes in the opacity caused by a strong magnetic field, which means that we see the inside rather than the surface of the granulation, and because the temperature is higher inside the granulation cells than at their surfaces the faculaes appear brighter than their surroundings (Keller et al. 2004). The carateristic timescale of the faculae is shorter than the timescale of the granulation cells because the faculaes only sample the edges of the granulation cells.

Following Harvey (1985) we modelled the smoothed PSD in the range [200-8000] $\mu \mathrm{Hz}$ with a background model containing two components plus the photon-noise contribution $(c)$ that dominates the PSD at high frequency, i.e.

$$
\operatorname{PSD}(v)=c+\frac{4 \sigma_{\mathrm{gran}}^{2} \tau_{\mathrm{gran}}}{1+\left(2 \pi \tau_{\mathrm{gran}} v\right)^{a_{\mathrm{gran}}}}+\frac{4 \sigma_{\mathrm{facu}}^{2} \tau_{\mathrm{facu}}}{1+\left(2 \pi \tau_{\mathrm{facu}} \nu\right)^{a_{\mathrm{facu}}}} .
$$

Here, $\sigma_{\text {gran }}$ and $\sigma_{\text {facu }}$ are the amplitudes of the background signal of granulation and the second convective component or faculae respectively, $\tau$ is the timescale, and $a_{\text {gran }}$ and $a_{\text {facu }}$ are two constants. Because we are modelling a smoothed version of the PSD we can assume that the error between the model and the observations are normally distributed. We can therefore fit the model to the observations by means of least squares. Using the robust non-linear least-squares curve fitting IDL package MPFIT (http://www.physics.wisc.edu/ craigm/ $\mathrm{idl} / \mathrm{idl} . \mathrm{html}$ ) we obtained a granulation timescale of $383 \pm$ $28 \mathrm{~s}$ and a second component with a timescale of $113 \pm 51 \mathrm{~s}$.

We then assumed that the second bump around $1000 \mu \mathrm{Hz}$ is caused by $\mathrm{p}$ modes. Thus, for the background fitting, we used the first two terms of Eq. (2) for the background fitting - the photon noise and one Harvey model component - and fitted a Gaussian function to the second bump. This led to a very similar fit compared to the previous one, with the same parameters for the granulation timescale (see Fig. 9). Consequently, both assumptions could be correct and we cannot distinguish which is the best one at this stage of the analysis.

\subsection{Estimating the mean large separation}

In order to determine whether HD 170987 presents solar-like oscillations, we applied several methods. One of them consists of searching for the signature of the mean large separation

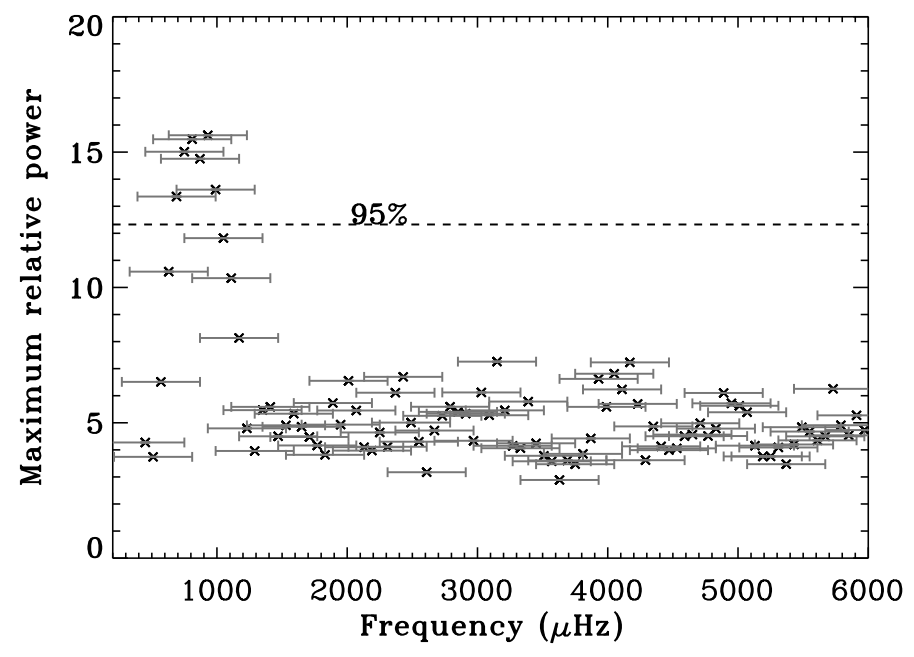

Fig. 10. Maximum relative power of the highest peak in the PS2 around half the large separation as a function of the central frequency of the sliding box taken in the PSD. The dashed line represents the threshold corresponding to a 95\% confidence level. The horizontal error bars represent the boxes in which the PSD has been sliced $(600 \mu \mathrm{Hz}$ in this case).

of a solar-like oscillating signal in the autocorrelation of the timeseries. As proposed by Roxburgh \& Vorontsov (2006), this autocorrelation is calculated as the Fourier spectrum of the windowed Fourier spectrum, in a refined application of the WienerKhinchine theorem. Roxburgh (2009) subsequently used a narrower window to look for the variation of the large separation with frequency.

Mosser \& Appourchaux (2009) have shown how to optimize the method and determine its reliability. They have scaled the autocorrelation function according to the noise contribution to statistically test its significance. When the envelope autocorrelation function (EACF) gives a signal above a defined threshold level, the null hypothesis can be rejected, and a reliable large separation can be derived. For a blind analysis of the mean value, $\langle\Delta v\rangle$, of the large separation and a rejection of the null hypothesis at the level $1 \%$, the threshold level is fixed to 8 . This mean value is determined in a frequency range centred on the frequency $v_{\mathrm{EACF}}$ where the EACF reaches its maximum amplitude, with a filter of width equal to the full-width-at-half-maximum of the mode envelope. Note that $v_{\mathrm{EACF}}$ is close to $v_{\max }$, where the mode envelope reaches its maximum amplitude, but they are not equal. The difference between $v_{\mathrm{EACF}}$ and $v_{\max }$ is due to the varying mode lifetime.

The method has shown to be efficient in low signal-to-noise cases, such as the CoRoT target HD 175726 (Mosser et al. $2009 \mathrm{~b}$ ) or in the K1V target with a height-to-background ratio $\mathcal{R}$ as low as $2.5 \%$ (Gaulme et al. 2009). The ratio $\mathcal{R}$ measures the maximum smoothed height of the modes compared to the background. The star HD 170987 is also a challenging target, with $\mathcal{R} \simeq 4 \%$. With an optimised filter of $580 \mu \mathrm{Hz}$ the EACF peaks at the frequency $930 \mu \mathrm{Hz}$ at a value of 19.2 for HD 170987 and gives a mean large separation of $55.9 \pm 0.2 \mu \mathrm{Hz}$.

Other methods have been employed and yielded a mean large separation between 54 and $56 \mu \mathrm{Hz}$ (Hekker et al. 2010a; Mathur et al. 2010).

Indeed, we calculated the power spectrum (PS) of the power spectrum (PS2) between 100 and $10000 \mu \mathrm{Hz}$ with the method described in Mathur et al. (2010). We selected the highest peak and assumed that it corresponds to half the mean large 

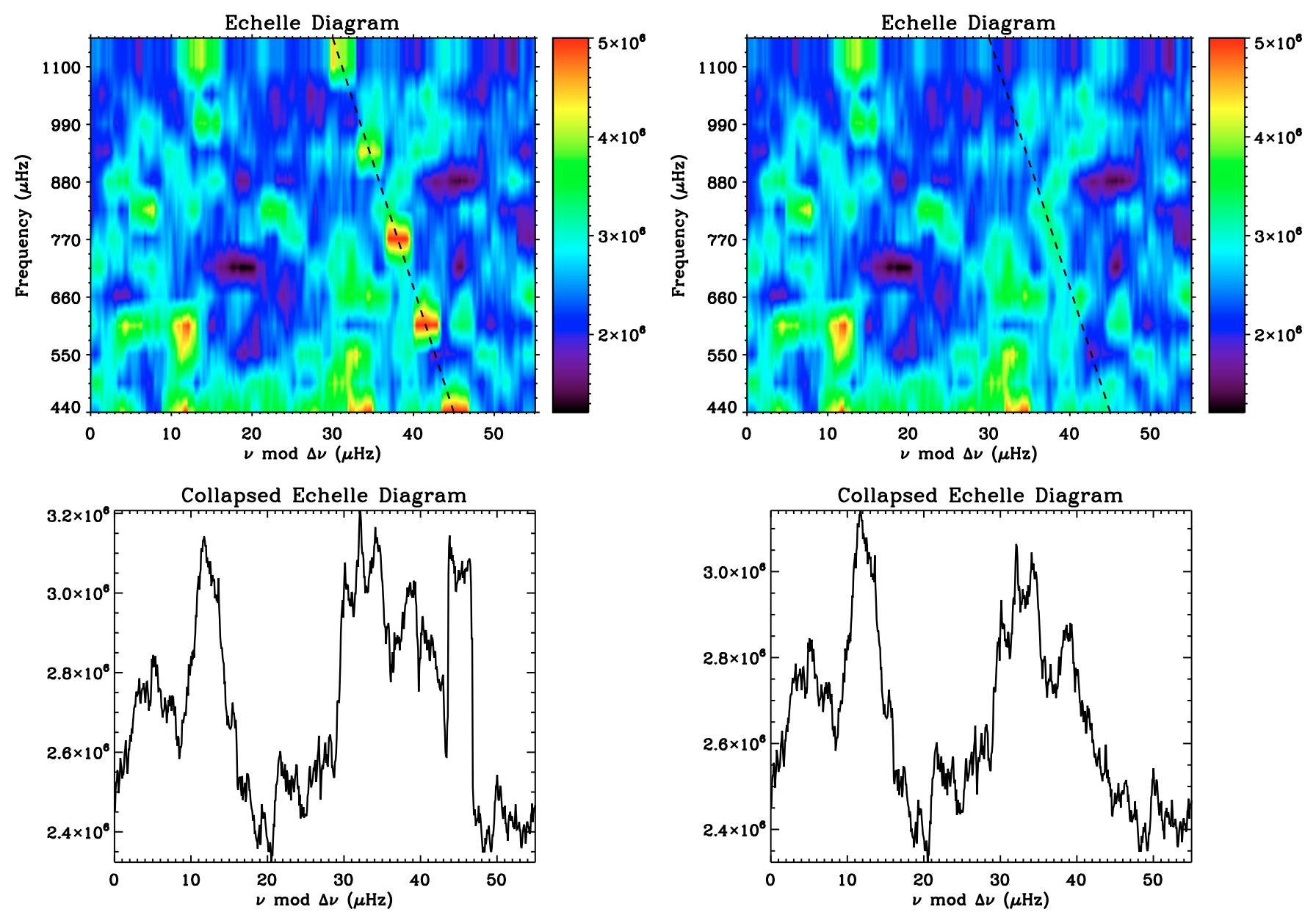

Fig. 11. Echelle diagrams obtained with the inpainted PSD (left panel) and the cleaned PSD (right panel) as explained in the text. They are computed with a folding frequency of $55 \mu \mathrm{Hz}$ in the region [440-1200] $\mu \mathrm{Hz}$ where the excess p-mode power was found. Bottom panels are the integrated (collapsed) échelle diagrams along the vertical axis. The dashed line represents the places where the orbital harmonics are situated.

separation. Then we cut the original PSD in boxes of $600 \mu \mathrm{Hz}$, shifted every $60 \mu \mathrm{Hz}$ and computed the PS2 of each box looking for the highest peak close to the originally found value. In Fig. 10 we present the power of the highest peak found in each box (normalised by the $\sigma$ of the PS2). The horizontal error bars mark the frequency range covered by each box. We obtained that the frequency-range of the $\mathrm{p}$ modes is [400-1200] $\mu \mathrm{Hz}$ with $\langle\Delta v\rangle=55.2 \pm 0.8 \mu \mathrm{Hz}$ with $95 \%$ confidence level.

Finally, we took into account that $\Delta v$ depends on frequency and computed the PS2 of a power spectrum with a stretched frequency axis (Hekker et al. 2010a). The stretching was performed in a way to produce an equidistant pattern of peaks on the stretched, as opposed to the original, frequency axis. This was done by applying a quadratic correction to the axis values. This allowed us to measure the linear variation of the $\Delta v$ with frequency (which are in themselves first differences of frequencies). The PS2 of the stretched power spectrum will therefore show a stronger (more prominent) signature of $\Delta v$ than the PS2 of the original spectrum. In the PS2 we determined the position of the $\Delta v / 2$ and $\Delta v / 4$ features and computed the Bayesian posterior probability of the features that would be due to noise. With these probabilities we then computed the posterior weighted centroids of the features. The interval with a probability of the feature that is not due to noise higher than $68.27 \%$, i.e., $1 \sigma$ in a Gaussian distribution, was used as the uncertainty interval. For this star we could only find a $\Delta v / 2$ feature in the PS2, which was used to determine $\langle\Delta v\rangle$.
We note that a large separation around $55 \mu \mathrm{Hz}$ is very close to the third harmonic of the orbit $(53.9 \mu \mathrm{Hz})$, which is still present in the inpainted data. Therefore we repeated the analyses described before for a cleaned version of the power spectrum in which we removed by hand the remaining peaks of the orbital harmonics, substituting the affected bins by a local average of the PSD (see Fig. 11). The results remained unchanged, which means that the pattern is not a consequence of an instrumental artifact.

With the large separation previously computed, we could calculate the so-called échelle diagram (Grec et al. 1983). To do so, we folded the power spectrum using a large separation of $55 \mu \mathrm{Hz}$ in the region where the p-mode excess was found [4401200] $\mu \mathrm{Hz}$. To reduce the dispersion of points in the spectrum we first smoothed it by a boxcar function of 40 points $(3.1 \mu \mathrm{Hz})$. The result is shown in the top left panel of Fig. 11. This diagram is dominated by the harmonics of the orbit at multiples of $161.7 \mu \mathrm{Hz}$, which are seen as an inclined line of points starting at $48 \mu \mathrm{Hz}$. If we used the cleaned version of the PSD (where we removed most of the peaks related to the orbital harmonics) and did the same analysis (see top right panel in Fig. 11), two nearly vertical ridges clearly appear in the diagram. Indeed, if we integrated (collapse) the échelle diagram in the vertical direction we obtained two well separated ridges (see bottom panels in Fig. 11). We can tentatively assign the left-hand ridge with even-l modes and the right-hand one to the odd ones knowing that the signal-to-noise ratio is not enough to go beyond that. 


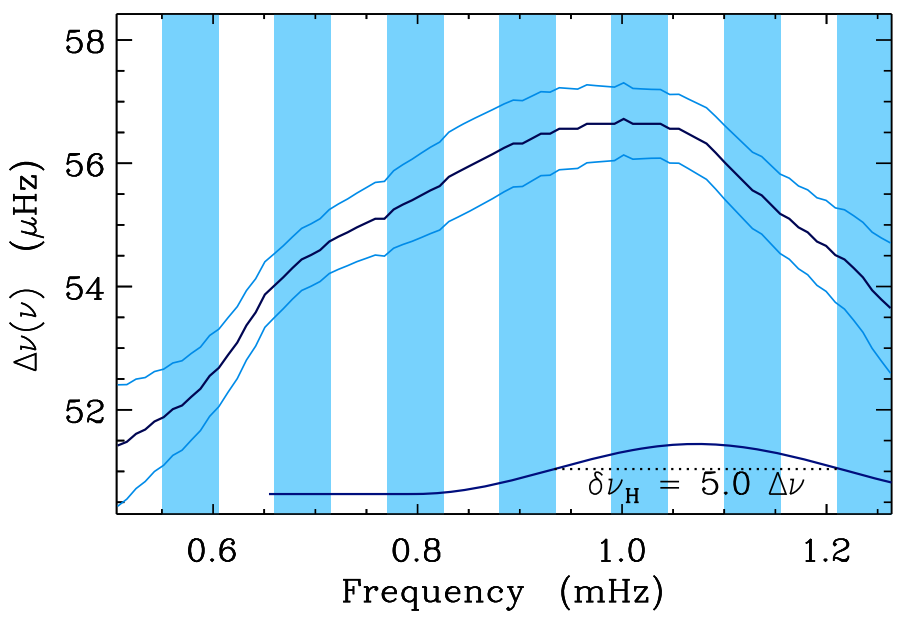

Fig. 12. Variation of the large separation with frequency. 1- $\sigma$ error bars are indicated. The extent of the Hanning filter used by the EACF is indicated in the lower-right corner, the dotted line measuring the fullwidth-at-half-maximum of the filter, here equal to 5 times the mean large separation. The width of the white and grey vertical regions is equal to $\langle\Delta v\rangle$.

The values of the mean large separation found by the different groups agree within their error bars, and in the following sections we will take $\langle\Delta v\rangle=55.2 \pm 0.8 \mu \mathrm{Hz}$ for the range [400$1200] \mu \mathrm{Hz}$. It is important to note that the error bar given here is the internal error of one single method and does not take into account the dispersion in the results from the different pipelines and the slightly different ranges used in their computations.

\subsection{Variation of the large separation}

When calculated with a filter narrower than the mode envelope, the EACF makes it possible to investigate the variation $\Delta v(v)$ of the large separation with frequency. With a filter width equal to 5 times the mean large separation, we tested the variation at a large scale: the large separation increases with frequency at a rate $\mathrm{d} \Delta v / \mathrm{d} n$ of about $0.55 \pm 0.15 \mu \mathrm{Hz}$ per radial order, reaches its maximum at $980 \mu \mathrm{Hz}$ and then decreases (Fig. 12).

With a filter width equal to twice the mean large separation we investigated the rapid variation of the large separation. The number of independent measurements allows us to show the modulation of the large separation (Fig. 13). The threshold level is lower than for the mean large separation for our study of the large separation with frequency $\Delta v(v)$. This enabled us to search the large separation in a predetermined reduced interval compared to the blind analysis. Most of the values of the EACF with such a filter are above 4.6 , which corresponds to the $1 \%$ rejection level for the analysis with a $2-\langle\Delta v\rangle$ wide filter and a search in the frequency range allowing $20 \%$ variation with respect to the mean large separation. They give error bars lower than $1 \mu \mathrm{Hz}$, except in the ranges [790-900] $\mu \mathrm{Hz}$ and [1180-1280] $\mu \mathrm{Hz}$. The EACF values are high enough to enable an unambiguous detection of the modulation. The period $W$ of this modulation, close to $200 \mu \mathrm{Hz}$, could be caused primarily by the He II ionisation zone or it could be related to the internal phase shifts. Following Monteiro \& Thompson (2005), we infer the acoustic depth $\tau \simeq 1 / 2 W$ of this region in the range $\tau=2300-2700 \mathrm{~s}$.

This analysis shows a peculiar behaviour in the range [790900] $\mu \mathrm{Hz}$. One would expect high values of the EACF in this range close to $v_{\max }$, which is not the case. Because the variations of $\Delta v(v)$ are not more pronounced in this region than in others,

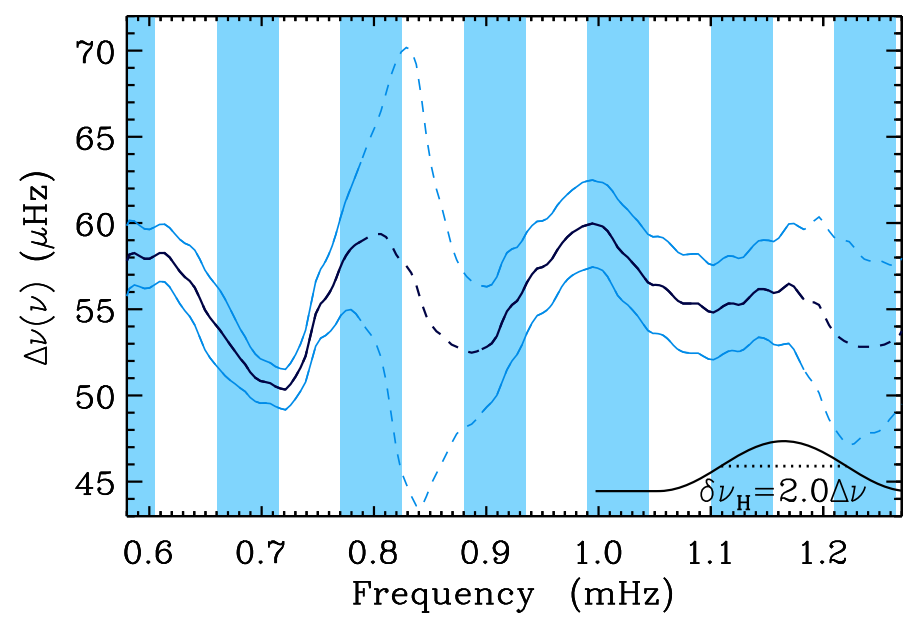

Fig. 13. Same as Fig. 12, but with a narrow filter, corresponding to twice the mean large separation. The thin lines correspond to frequency ranges where the EACF is below the threshold level for a null hypothesis rejection at the $1 \%$ level. The dashed lines are the regions perturbed by the orbital harmonics.

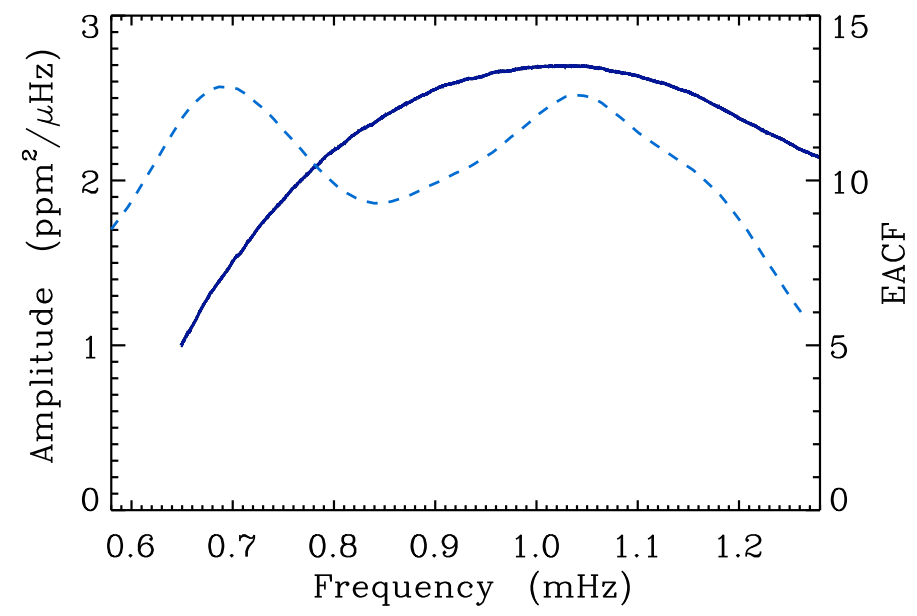

Fig. 14. Comparison of the EACF (dashed line), estimated with a 5- $\langle\Delta v\rangle$ large filter, and the bolometric mode amplitude expressed in ppm (continuous line).

one can explain the low values of the EACF either by a perturbation caused by a mixed mode or by a low value of the mode lifetime. A mixed mode is quite unlikely though: a mode with a much longer lifetime than normal $\mathrm{p}$ mode should appear with a large amplitude, which is not the case. Thus, one has to favour the hypothesis of the varying mode lifetime. The comparison of the EACF with the bolometric amplitude per radial mode (see Sect. 5.4) indicates a strong increase of the mode lifetime below $850 \mu \mathrm{Hz}$ and a strong decrease above $1100 \mu \mathrm{Hz}$ (Fig. 14). This behaviour agrees with the measurement made in a similar star with a very close large separation, such as HD 49385 (Deheuvels et al. 2010), in spite of a different effective temperature. However, it is not yet possible to quantify the lifetime.

\subsection{Maximum bolometric amplitude}

To compute the maximum bolometric amplitude per radial mode we needed to smooth the p-mode hump and correct from the background in this frequency range. To do so, we used different methods (see for an extensive explanation Kjeldsen et al. 2008; Hekker et al. 2010a; Mathur et al. 2010). Then to correct 
from the instrumental response function we used the method developed by Michel et al. (2009). Thus, we obtained a bolometric amplitude per radial mode of $A_{\text {bol, }=0}=2.7 \pm 0.2 \mathrm{ppm}$ at $\sim 1070 \mu \mathrm{Hz}$. However, among the different teams and depending on the method and the way we fitted the background, the value for the maximum amplitude per radial mode varied within the range 2.4 to $2.9 \mathrm{ppm}$, indicating that the error bar of $0.2 \mathrm{ppm}$ could be underestimated. So we estimated a new value for the error of $0.6 \mathrm{ppm}$, which was derived from the scatter of the smoothed power spectrum about the background fit outside the oscillation range (Huber et al. 2009).

The top panel of Fig. 15 shows the comparison of the amplitude found for HD 170987 with the published values of four other CoRoT solar-like targets (Michel et al. 2008; Mosser et al. $2009 \mathrm{~b})$ as a function of $v_{\max }$. The bottom panel shows the amplitudes compared to theoretical values for each star calculated using the $(L / M)^{s} T_{\text {eff }}^{-0.5}$ scaling relation from Eq. (3) of Kjeldsen $\&$ Bedding (1995) with $s=0.7$ (Samadi et al. 2007b). It can be seen that while in all cases the amplitudes are systematically about $30 \%$ lower than the theoretical values, the discrepancy appears to be significantly larger for HD 170987 ( 50\%). We note that the error bars are larger for HD 170987 due to the rather large uncertainty of the parallax $(\sim 10 \%)$. In both cases, the discrepancy is well inside $3-\sigma$.

\section{Inferring global stellar parameters}

Using $\langle\Delta v\rangle=55.2 \pm 0.8 \mu \mathrm{Hz}, f_{\min }=400 \mu \mathrm{Hz}, f_{\max }=$ $1200 \mu \mathrm{Hz}$ (see Sect. 5.1 and 5.2), and the spectroscopic information $\log g=4.20 \pm 0.14 \mathrm{dex},[M / H]=-0.20 \pm 0.15 \mathrm{dex}$ and $T_{\text {eff }}=6540 \pm 80 \mathrm{~K}$ (see Sect. 2), we compared these values with stellar models to determine the radius and the mass of HD 170987. The stellar models that we used are the Aarhus Stellar Evolution Code (ASTEC) coupled with an adiabatic pulsation code (ADIPLS) (Christensen-Dalsgaard 2008a,b). These codes need as input the stellar parameters of mass, age, chemical composition, and mixing-length parameter, and return stellar observables $B_{i}$, such as radius, effective temperature, and the oscillation mode frequencies.

The parameters that best describe the observables are obtained by minimising a $\chi^{2}$ function,

$\chi^{2}=\sum_{i=1}^{M}\left(\frac{y_{i}-B_{i}}{\epsilon_{i}}\right)^{2}$,

where $y_{i}$ and $\epsilon_{i}$ are the $i=1,2, \ldots, M$ observations and errors. Here, we have $M=4$. The Levenberg-Marquardt algorithm is used for the optimization, and this incorporates derivative information to guess the next set of parameters that will reduce the value of $\chi^{2}$. Naturally, an initial guess of the parameters is needed and these are obtained from a small grid of stellar evolution tracks.

Because there are few observations and just as many parameters, there are inherent correlations between mass, age and chemical composition. To help avoid local minima problems, we minimised the $\chi^{2}$ function beginning at several initial guesses of the parameters (mainly varying in mass and age). These initial guesses were estimated from the grids. We obtained several sets of parameters with a corresponding $\chi^{2}$ value that match the observations as best as possible. Choosing the models whose $\chi^{2}$ values fall below $3.9^{2}$ (assuming that the errors are Gaussian and that we aim for a $99 \%$ probability), the radius and mass were determined by calculating the average value of each, and the uncertainty is given by the dispersion in the values divided by 6 (see
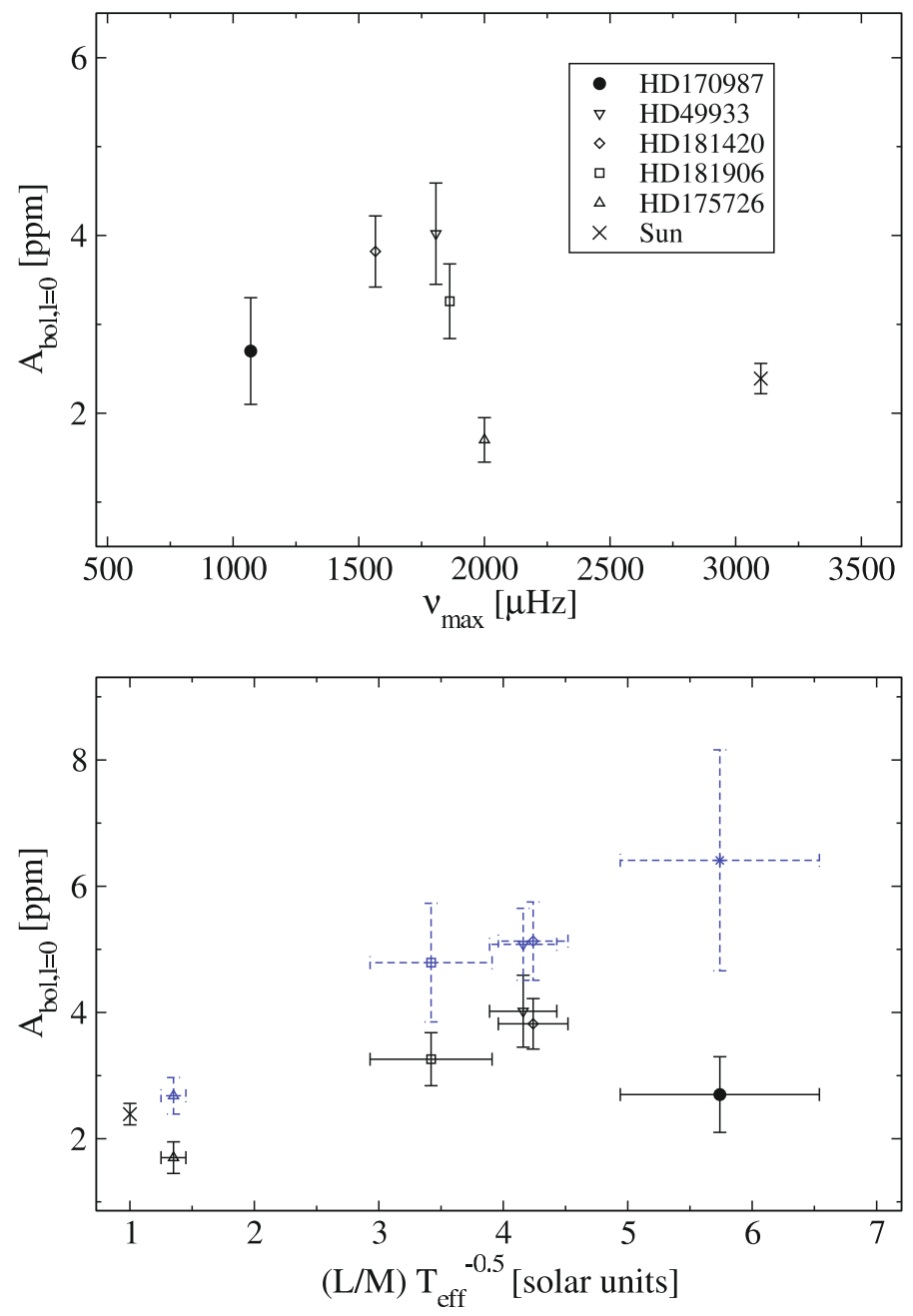

Fig. 15. Top panel: Maximum bolometric amplitude per radial mode versus $v_{\max }$ for published CoRoT F-stars (open symbols), the Sun (star symbol) and HD 170987 (filled circle). Bottom panel: Maximum bolometric amplitude per radial mode versus stellar parameters. Black symbols are the observed values, and blue symbols theoretical values using $(L / M)^{0.7}$. The symbol types are the same as in the top panel.

Table 2. Stellar parameters from modelling.

\begin{tabular}{lll}
\hline \hline Parameter & Value & Error \\
\hline Radius $\left(R_{\odot}\right)$ & 1.960 & 0.046 \\
Mass $\left(M_{\odot}\right)$ & 1.43 & 0.05 \\
Age $(\mathrm{Gyr})$ & 2.4 & - \\
\hline
\end{tabular}

Mathur et al. 2010, for details). We estimated the age of the star by simply matching the "fitted mass" with the closest age from the model parameter results, we therefore have no quantitative measure of its uncertainty, leading to a very preliminary value. The results are given in Table 2. The estimate of the age implies a star coming close to the end of the main-sequence phase.

\section{Discussion}

Thanks to spectroscopic observations from NARVAL, we were able to estimate the effective temperature as well as the chemical abundances of HD 170987, which show that this star has a low metallicity $(-0.15 \pm 0.06 \mathrm{dex})$ and a high abundance of lithium.

The surface light elements abundances could give us an interesting clue about the internal rotation of HD 170987. The effective temperature of $6540 \mathrm{~K}$ sets this star in the so-called 
lithium dip where both ${ }^{7} \mathrm{Li}$ and ${ }^{9} \mathrm{Be}$ are depleted (Boesgaard \& King 2002). This abundance dip is most likely related to rotation and observations show that depletion and rotation are correlated (Boesgaard 1987). Talon \& Charbonnel (2003) explained the cooler side of the lithium dip (from 6500 to $6200 \mathrm{~K}$ ) by invoking an increasing efficient transport of the internal angular momentum through gravity waves when the mass decreases from $\approx 1.4$ to $\approx 1.2 M_{\odot}$. For the more massive stars differential rotation in the interior leads to shear instabilities: the radiative envelope is mixed deeply and light elements are destroyed through proton capture. For the less massive stars there is less differential rotation and less shear instabilities. Thus no (or far less) depletion occurs.

The lithium dip concerns stars with an effective temperature in the range 6400 to $6850 \mathrm{~K}$. At Hyades age this star should already have exhibited a depletion of about 0.5 dex in ${ }^{7} \mathrm{Li}$ (Boesgaard \& King 2002). However HD 170987 seems much older than Hyades and shows no such depletion: if we combine the current solar ${ }^{7} \mathrm{Li}$ abundance (Asplund et al. 2009) and the relative abundance of HD170987 to the Sun given above we obtain $\mathrm{A}\left({ }^{7} \mathrm{Li}\right) \approx 2.9 \mathrm{dex}$. This value is very close to the fraction of lithium in the material out of which Population I stars form: 3.3 to 3.2 dex. Thus the abundance of ${ }^{7} \mathrm{Li}$ in the atmosphere of HD 170987 directly supports the absence of deep mixing and indirectly supports the absence of differential rotation with depth. At least if some internal differential rotation exists it is not sufficient to induce deep mixing.

As confirmed by our analysis, HD 170987 is remarkably similar to Procyon, a prime target for previous asteroseismic studies using ground-based spectroscopy and space-based photometry. For the latter, observations by the MOST satellite resulted in a null-detection of p-modes (Matthews et al. 2004), leading to conclusions that were later critically discussed by Bedding et al. (2005). These observations were followed by WIRE photometry (Bruntt et al. 2005) and higher precision MOST data collected in 2007 (Guenther et al. 2008), both showing power excess around the expected p-mode location of $1 \mathrm{mHz}$, while velocity observations revealed a clear $\mathrm{p}$-mode structure (see, e.g., Arentoft et al. 2008; Bedding et al. 2010b). Convection models by Guenther et al. (2008) showed that for Procyon, as opposed to the Sun, the granulation timescale can coincide with the location of pulsation frequencies. The resulting interaction with the acoustic oscillations causing short mode lifetimes were identified as possible difficulties for detecting clear peak spacings in the photometry of Procyon-like stars. Given the similarity of HD 170987 to Procyon, it is tempting to speculate that a similar mechanism is in part responsible for the low visibility of the pulsation modes in the CoRoT photometry of HD 170987. Alternate mechanisms which could partly explain the low signal include the low metallicity (see Samadi et al. 2010a) or the fact that some of the light is diluted by a possible binary companion.

\section{Conclusions}

We analysed the 149 days of the light curve of the CoRoT target HD 170987, allowing us to determine its global parameters. We found that the rotation period of the star is around 4.3 days and the $v \sin i$ of $19 \mathrm{~km} \mathrm{~s}^{-1}$, leading to an inclination angle $i$ of $\sim 50^{\circ}{ }_{-13}^{+20}$. By fitting the background, we obtained that the timescale for the granulation is around $383 \pm 28 \mathrm{~s}$, either assuming that we have some contribution of faculae or of modes.

We confirm that the power excess between 400 and $1200 \mu \mathrm{Hz}$ is due to acoustic modes, which are characterised by a mean large separation $\langle\Delta v\rangle=55.2 \pm 0.8 \mu \mathrm{Hz}$ with more than $95 \%$ of probability and a posteriori probability of $68 \%$. With the EACF method we found $\langle\Delta v\rangle=55.9 \pm 0.2 \mu \mathrm{Hz}$ in a higher frequency range [500-1250] $\mu \mathrm{Hz}$ with a $1 \%$ rejection. However, we recognise that all these confidence levels are dependent on our assumption that our spectrum is dominated by white noise statistics. It may therefore be that we should be less confident as there could be other sources of noise embedded in the signal. However, at this level the detection seems to be unambiguous. Moreover, this value agrees with the expected one derived from the stellar fundamental parameters, which gives $52.7 \pm 4 \mu \mathrm{Hz}$, the large error bar is due to the large uncertainty on the parallax. Now, thanks to the detection of this pattern, we can be confident that the bump observed around $1000 \mu \mathrm{Hz}$ is very likely related to acoustic modes.

We characterise the power excess with a maximum amplitude of $2.7 \pm 0.6 \mathrm{ppm}$ at $\sim 1070 \mu \mathrm{Hz}$, where the uncertainty on the amplitude has been obtained by taking into account the scatter of the smoothed power spectrum about the background fit outside the oscillation range.

Because of the low signal-to-noise ratio, we cannot fit the acoustic modes individually. However, from the global seismic parameters found and the results form the NARVAL spectrohraph, we estimated that HD 170987 has a mass, $M=1.43 \pm$ $0.05 M_{\odot}$, a radius, $R=1.96 \pm 0.046 R_{\odot}$, and an age $\sim 2.4$ Gyr.

Further studies are needed to have a better comprehension of the wave propagation in the stellar atmosphere of stars located at this position in the HR diagram. It would also enable us to understand why the oscillation-amplitudes measured in intensity fluctuations are so low.

Acknowledgements. The authors thank S. Pires and J.L. Stark for their useful comments and discussions on the inpaining methods applied in this paper as well as T.R. Bedding for his useful comments. D.S. acknowledges Australian Research Council. J.B. acknowledges the ANR Siroco of the French agency for research. This work has been partially supported by: the CNES/GOLF grant at the Service d'Astrophysique (CEA/Saclay) and the grant PNAyA200762650 from the Spanish National Research Plan. This work benefited from the support of the International Space Science Institute (ISSI), through the workshop programme award. It was also partly supported by the European Helioand Asteroseismology Network (HELAS), a major international collaboration funded by the European Commission's Sixth Framework Programme. I.W.R., G.A.V., S.H., W.J.C., and Y.E. acknowledge support from the UK Science and Technology Facilities Council (STFC).

\section{Appendix A: Inpainting interpolation of the light curve}

"Inpainting" refers to methods that interpolate the missing information using some priors on the solution. The method we applied to HD 170987 uses a prior of sparsity and was introduced by Elad et al. (2005). It has already been applied to various fields of astrophysics, such as weak lensing (Pires et al. 2009) and recently to asteroseismic timeseries by Sato et al. (in prep.). It assumes that there exists a dictionary $\Phi-$ which in our case is a discrete cosine transform (DCT) - where the complete data are sparse and the incomplete data are less sparse. It means that there exists a representation $\alpha=\Phi^{T} X$ of the signal $X$ in the dictionary $\Phi$ where most coefficients $\alpha_{i}$ are close to zero. Let $X$ be the ideal complete timeseries, $Y$ the observed timeseries and $M$ the window function or mask (i.e. $M_{i}=1$ where there is a valid data point and $M_{i}=0$ elsewhere). Thus we have $Y=M X$. Inpainting consists in recovering $X$ knowing $Y$ and $M$. The solution is obtained by minimising the following equation

$\min _{X}\left\|\Phi^{T} X\right\|_{1}$ subject to $\sum_{i}(Y-M X)^{2} \leq \sigma$ 
where $\sigma$ stands for the noise standard deviation and where we use a pseudo norm, with $\|z\|_{1}=\sum_{k}\left|z_{k}\right|$. To minimise this expression, an iterative algorithm was used.

In this study, we built the mask $M$ to remove those points which are affected by the SAA and also all the other points that were already linearly interpolated, according to the status flag provided in the $\mathrm{N} 2$ files.

Most of the gaps in the CoRoT timeseries have a typical timescale of several minutes. It is possible to interpolate these typical gaps by using an inpainting algorithm based on a DCT decomposition which is localised in a short timescale. However, there are also a few longer gaps which have a timescale of several hours in the timeseries. The inpainting algorithm needs to take into account this large variation of the scale of the gaps. To do that, we replaced the simple DCT decomposition by a multiscale discrete cosine transform decomposition. With this transform, we first decomposed the light curve into different scales using a wavelet transform. Then the inpainting of each scale allowed us to interpolate the gaps of different timescales. The PSD of the inpainted timeseries is shown in Fig. 5 (bottom). The improvement is clearly visible by a reduction of the amplitude of the orbital harmonics by more than $90 \%$ in many cases, as well as the disappearance of the daily modulation except for the first orbital harmonic (see the inset of Fig. 5 (bottom) corresponding to the second orbital harmonic, in which the main peak is reduced by $95 \%$ and no signature of the daily modulation is seen).

\section{References}

Allende Prieto, C., Asplund, M., López, R. J. G., \& Lambert, D. L. 2002, ApJ, 567,544

Appourchaux, T., Michel, E., Auvergne, M., et al. 2008, A\&A, 488, 705

Arentoft, T., Kjeldsen, H., Bedding, T. R., et al. 2008, ApJ, 687, 1180

Asplund, M., Grevesse, N., Sauval, A. J., \& Scott, P. 2009, ARA\&A, 47, 481

Auvergne, M., Bodin, P., Boisnard, L., et al. 2009, A\&A, 506, 411

Baglin, A., Michel, E., Auvergne, M., \& The COROT Team. 2006, in Proc.

SOHO 18/GONG 2006/HELAS I, Beyond the spherical Sun, ESA SP, 624

Ballot, J., García, R. A., \& Lambert, P. 2006, MNRAS, 369, 1281

Ballot, J., Appourchaux, T., Toutain, T., \& Guittet, M. 2008, A\&A, 486, 867

Barban, C., Deheuvels, S., Baudin, F., et al. 2009, A\&A, 506, 51

Bedding, T. R., \& Kjeldsen, H. 2007, Commun. Asteroseismol., 150, 106

Bedding, T. R., Kjeldsen, H., Bouchy, F., et al. 2005, A\&A, 432, L43

Bedding, T. R., Huber, D., Stello, D., et al. 2010a, ApJ, 713, L176

Bedding, T. R., Kjeldsen, H., Campante, T. L., et al. 2010b, ApJ, 713, 935

Boesgaard, A. M. 1987, PASP, 99, 1067

Boesgaard, A. M., \& King, J. R. 2002, ApJ, 565, 587

Borucki, W., Koch, D., Batalha, N., et al. 2009, IAU Symp., 253, 289

Bruntt, H. 2009, A\&A, 506, 235

Bruntt, H., Bikmaev, I. F., Catala, C., et al. 2004, A\&A, 425, 683

Bruntt, H., Kjeldsen, H., Buzasi, D. L., \& Bedding, T. R. 2005, ApJ, 633, 440

Bruntt, H., Suárez, J. C., Bedding, T. R., et al. 2007, A\&A, 461, 619

Bruntt, H., De Cat, P., \& Aerts, C. 2008, A\&A, 478, 487

Bruntt, H. Bedding, T. R., Quirion, P.-O., et al. 2010, MNRAS, 405, 1907

Chaplin, W. J., Appourchaux, T., Elsworth, Y., et al. 2010, ApJ, 713, L169

Christensen-Dalsgaard, J. 2004, Sol. Phys., 220, 137

Christensen-Dalsgaard, J., \& Houdek, G. 2009, [arXiv : 0911.4629]

Creevey, O. L. 2008, [arXiv:0810.2440], unpublished

Deheuvels, S., \& Michel, E. 2009, Ap\&SS, 241

Deheuvels, S., Bruntt, H., Michel, E., et al. 2010, A\&A, 515, A87

Dommanget, J., \& Nys, O. 2002, VizieR Online Data Catalog, 1274, 0
Donati, J., Semel, M., Carter, B. D., Rees, D. E., \& Collier Cameron, A. 1997, MNRAS, 291, 658

Flower, P. J. 1996, ApJ, 469, 355

Fröhlich, C., Romero, J., Roth, H., et al. 1995, Sol. Phys., 162, 101

Fuhrmann, K., Pfeiffer, M., Frank, C., Reetz, J., \& Gehren, T. 1997, A\&A, 323, 909

García, R. A., Régulo, C., Samadi, R., et al. 2009, A\&A, 506, 41 Gaulme, P., Appourchaux, T., \& Boumier, P. 2009, A\&A, 506, 7 Gizon, L., \& Solanki, S. K. 2003, ApJ, 589, 1009

Grec, G., Fossat, E., \& Pomerantz, M. A. 1983, Sol. Phys., 82, 55 Grevesse, N., Asplund, M., \& Sauval, A. J. 2007, Space Sci. Rev., 130, 105 Guenther, D. B., Kallinger, T., Gruberbauer, M., et al. 2008, ApJ, 687, 1448 Gustafsson, B., Edvardsson, B., Eriksson, K., et al. 2008, A\&A, 486, 951 Harvey, J. 1985, in Future Missions in Solar, Heliospheric \& Space Plasma Physics, ed. E. Rolfe, \& B. Battrick, ESA SP, 235, 199

Hekker, S., Broomhall, A., Chaplin, W. J., et al. 2010a, MNRAS, 402, 2049 Hekker, S., Debosscher, J., Huber, D., et al. 2010b, ApJ, 713, L187

Hinkle, K., Wallace, L., Valenti, J., \& Harmer, D. 2000, Visible and Near Infrared Atlas of the Arcturus Spectrum 3727-9300 A (San Francisco: ASP)

Holdschneider, M., Kronland-Martinet, R., Morlet, J., \& Tchamitchian, P. 1989, in Wavelets, ed. J. Combes (Springer-Verlag Berlin), 286

Holmberg, J., Nordström, B., \& Andersen, J. 2007, A\&A, 475, 519

Huber, D., Stello, D., Bedding, T. R., et al. 2009, Commun. Asteroseismol., 160, 74

Keller, C. U., Schüssler, M., Vögler, A., \& Zakharov, V. 2004, ApJ, 607, L59

Kjeldsen, H., \& Bedding, T. R. 1995, A\&A, 293, 87

Kjeldsen, H., Bedding, T. R., Arentoft, T., et al. 2008, ApJ, 682, 1370

Kupka, F., Piskunov, N., Ryabchikova, T. A., Stempels, H. C., \& Weiss, W. W. 1999, A\&AS, 138, 119

Ludwig, H., Samadi, R., Steffen, M., et al. 2009, A\&A, 506, 167

Mathis, S. 2009, A\&A, 506, 811

Mathur, S., Jimenez-Reyes, S. J., \& Garcia, R. A. 2008, in Proc. of GONG 2008/SOHO XXI, Solar-stellar dynamos as revealed by helio- and asteroseismology

Mathur, S., García, R. A., Régulo, C., et al. 2010, A\&A, 511, A46

Matthews, J. M., Kusching, R., Guenther, D. B., et al. 2004, Nature, 430, 51

Michel, E., Baglin, A., Auvergne, M., et al. 2008, Science, 322, 558

Michel, E., Samadi, R., Baudin, F., et al. 2009, A\&A, 495, 979

Monteiro, M. J. P. F. G., \& Thompson, M. J. 2005, MNRAS, 361, 1187

Mosser, B., \& Appourchaux, T. 2009, A\&A, 508, 877

Mosser, B., Baudin, F., Lanza, A. F., et al. 2009a, A\&A, 506, 245

Mosser, B., Michel, E., Appourchaux, T., et al. 2009b, A\&A, 506, 33

Piau, L., Turck-Chièze, S., Duez, V., \& Stein, R. F. 2009, A\&A, 506, 175

Pires, S., Starck, J., Amara, A., et al. 2009, MNRAS, 395, 1265

Press, W. H., Teukolsky, S. A., Vetterling, W. T., \& Flannery, B. P. 1992,

Numerical recipes in FORTRAN. The art of scientific computing, 2nd Ed. (Cambridge: University Press)

Rentzsch-Holm, I. 1996, A\&A, 312, 966

Roxburgh, I. W. 2009, A\&A, 506, 435

Roxburgh, I. W., \& Vorontsov, S. V. 2006, MNRAS, 369, 1491

Samadi, R. 2009 [arXiv: 0912 .0817], unpublished

Samadi, R., Fialho, F., Costa, J. E. S., et al. 2007a

[arXiv:astro-ph/0703354]

Samadi, R., Georgobiani, D., Trampedach, R., et al. 2007b, A\&A, 463, 297

Samadi, R., Ludwig, H., Belkacem, K., Goupil, M. J., \& Dupret, M. 2010a, A\&A, 509, A15

Samadi, R., Ludwig, H., Belkacem, K., et al. 2010b, A\&A, 509, A16

Sato, K. H., Garcia, R. A., Pires, S., et al. 2010 [ArXiv: 1003. 5178]

Stello, D., Chaplin, W. J., Bruntt, H., et al. 2009, ApJ, 700, 1589

Stello, D., Basu, S., Bruntt, H., et al. 2010, ApJ, 713, L182

Talon, S., \& Charbonnel, C. 2003, A\&A, 405, 1025

Torrence, C., \& Compo, G. P. 1998, Bull. Am. Meteorol. Soc., 79, 61

Valenti, J. A., \& Piskunov, N. 1996, A\&AS, 118, 595

van Leeuwen, F. 2007a, Hipparcos, the New Reduction of the Raw Data, ed.

F. van Leeuwen, Astrophys. Space Sci. Library, 350

van Leeuwen, F. 2007b, A\&A, 474, 653

Walker, G., Matthews, J., Kuschnig, R., et al. 2003, PASP, 115, 1023 